\title{
Caracterização do comportamento mecânico e microestrutural do PEAD aditivado com eletrodo de solda
}

\author{
Characterization of the mechanical and microstructural behavior of the HDPE added with welding \\ electrode
}

\author{
Ana Carolina Ferreira ${ }^{\dagger}$, Francine Pamponet ${ }^{\dagger}$ Gabriela Rolim ${ }^{\dagger}$, Julia Dutra Braz ${ }^{\dagger}$, Lívia Saboya \\ Ricardo de Freitas Cabral*
}

Como citar esse artigo. Ferreira, AC; Pamponet, F; Rolim, G; Braz, JD; Saboya, L; Cabral, RF. Caracterização do comportamento mecânico e microestrutural do PEAD aditivado com eletrodo de solda. Revista Teccen. 2019 Jan/Jun; 12 (1): 23-30.

\begin{abstract}
Resumo
O Polietileno é um polímero mais simples em relação à cadeia de polímeros, mundialmente é um dos mais produzidos e consumidos que torna o plástico mais comum não só pelas suas características químicas, mas também por ser um material mais barato. O polietileno de alta densidade (PEAD) é produzido a partir da polimerização do monômero eteno e também por isso pode ser conhecido como polietileno. Ele é resistente à alta temperatura, possui alta resistência à compressão e tração e moderada resistência ao impacto. Por ser um polímero com a densidade elevada é considerado especialmente por fabricação, diversos produtos, tais como embalagens, tanques, sacolas, por ser um material não reativo, atóxico e com boa resistência química têm excelentes propriedades mecânicas, físicas químicas e hidráulicas. O eletrodo de solda foi usado no material para conferir mais resistência ao material final, aumentando sua resistência e também analisar algumas variáveis que influenciam as características do PEAD. No presente trabalho, os corpos de prova foram analisados pelos ensaios de resistência àflexão, dureza Shore D e análise da microestrutura das amostras por microscopia óptica e microscopia eletrônica de varredura (MEV). O presente compósito apresentou valor médio de dureza de 60,816 SD e tensão máxima de $27 \mathrm{MPa}$. As fibras do compósito apresentaram desordenação, com fraca interação do material. Palavras-Chave: PEAD, Caracterização microestrutural, Propriedades mecânicas, Eletrodo de solda.
\end{abstract}

\begin{abstract}
Polyethylene is a more simple polymer in relation to polymer chain, worldwide is one of the most produced and consumed polymers that makes the plastic more common not just by its chemical features, but also for being a cheaper material. The high-density polyethylene (HDPE) is produced by polymerization of ethene monomer and because of that is also known as polyethylene, its features are the high temperature resistance, also tension, compression and traction high resistance, the plastic is the most rigid and has a moderate impact resistance. By being a polymer with increased density, it is considered specially by fabricating several products, such as packages, tanks, plastic bags, among others, because is a non-reactive, atoxic material and with good chemical resistance having excellent mechanical, physico-chemistry and hydraulic properties. The welding electrode was used on the material to impart more resistance to the final material, increasing its resistance and analyzing some variables which influences the features of HDPE. On present study, test bodies were analyzed on flexion test, hardness test, microstructure of the samples by optic microscopy and scanning electronic microscopy. The present composite presented hardness medium value of $60,816 \mathrm{SD}$ and maximum strength of $27 \mathrm{MPa}$.The fibers of the composite presented disorientation, with weak interaction of the material.

Keywords: HDPE, Microstructural characterization, Mechanical properties, Welding electrode.
\end{abstract}

\section{Introdução}

Os polímeros são compostos formados por macromoléculas onde pequenas partes que são chamadas monômeros. As macromoléculas são a base para a fabricação dos plásticos (Calister \& Rethwisch, 2012).

Os Polímeros estão presentes em uma variedade de objetos, como por exemplo: Sacolas, plásticos, para choques de automóveis, canos pra água, panelas antiaderentes, mantas, colas, tintas, chicletes (Canevarolo, 2007).

Segundo Mano(1999), alguns parâmetros internos a estrutura do polímero afetam acentuadamente seu comportamento mecânico, sendo esses: As informação das macromoléculas, a forma que elas se cristalizam,

Afiliação dos autores: † Mestrado Profissional em Materiais do Centro Universitário de Volta Redonda - UniFOA - Av. Paulo Erlei Alves Abrantes, 1325 - Três Poços, Volta Redonda - RJ - CEP 27240-560 - Brasil/Discentes do Mestrado Profissional em Materiais;

* Mestrado Profissional em Materiais do Centro Universitário de Volta Redonda - UniFOA - Av. Paulo Erlei Alves Abrantes, 1325 - Três Poços, Volta Redonda - RJ - CEP 27240-

560 - Brasil/Docente Responsável Doutor; e Centro Universitário Geraldo Di Biase - UGB - Rodovia Benjamin Lelpo, Km 11, Barra do Piraí - RJ - CEP 27101-090, Brasil/Docente Responsável Doutor

* Email para correspondência: ricardo.cabral@foa.org.br 
a presença de materiais aditivos, o nível de ligações cruzadas e o teor de umidade.

O objetivo desta pesquisa foi o de caracterizar amostras de PEAD reforçado com eletrodo de solda, por meio das suas propriedades mecânicas e morfológicas, nos ensaios de resistência à flexão e dureza Shore D e (MEV) e microscopia de luz (óptica), respectivamente.

\section{Materiais e Métodos}

\section{Moldagem por injeção}

Para a análise do PEAD foram feitos 2 corpos de prova. Para tal, foi realizada a injeção dos corpos de prova com o auxílio da injetora de plástico marca TSMP, onde foi adicionado $10 \%$ de pó de solda. O Polietileno foi aquecido durante aproximadamente 40 min até atingir uma temperatura de $280{ }^{\circ} \mathrm{C}$ e injetado em corpo de prova para realização do ensaio de dureza e de flexão.

\section{Preparação das amostras para microscopia de luz (óptica)}

\begin{abstract}
As amostras foram preparadas através de pequenas porções de cada corpo de prova e embutidas a frio, onde uma mistura de Resina Acrílica foi vertida em líquido auto-polimerizante. Após $5 \mathrm{~h}$, com a mistura já sólida, deformada e identificada no procedimento embutido, as amostras foram submetidas ao ensaio no microscópio óptico OptonTNM-07T-PLe foi utilizado a lente ocular no Microcomputador com o software ScopePhoto3.0 de $10 \mathrm{X}$ e as lentes objetivas de 1000X, 2000X e 4000X.
\end{abstract}

\section{Preparação das amostras para o MEV}

No MEV foi utilizado o microscópio da marca Hitachi modelo TM3000 e os ensaios foram realizados em ambientes de temperatura controlada de $20^{\circ} \mathrm{C}$. Os ensaios nas amostras embutidas e sem embutimento foram analisados logo após a sua metalização para a interação do feixe eletrônico. No processo de metalização alguns elétrons são absorvidos, para que as amostras se tornem condutoras. Foi usado um metalizador tipo EMITECH modelo K550X, com tempo de metalização de 2 min. Este processo de metalização foi realizado no laboratório de microscopia da UFF - campus Volta Redonda.

\section{Dureza Shore D}

Iniciou-se o teste de dureza no equipamento DUROMETRO Digital portátil tipo Shore D - TH 210, para a medição de dureza, foi realizado o teste nas 2 amostras, a fim de verificar a resistência de penetração de um corpo de prova duro, onde forem realizadas 5 medidas em cada corpo de prova.

\section{Resistência à flexão}

Após os corpos de prova serem submetidos ao teste de dureza, os mesmos foram levados ao teste de flexão para verificar o módulo de falhas a fim de avaliar as propriedades mecânicas, tensão e flecha de ruptura além do módulo de elasticidade.

O Teste foi realizado no equipamento EMIC modelo DL 10000. No ensaio do PEAD foi utilizada uma célula de carga de $5 \mathrm{kN}$ e uma taxa de carregamento de $30 \mathrm{~mm} / \mathrm{min}$. O Objetivo deste ensaio foi determinar o módulo de elasticidade e módulo de resistência à flexão.

\section{Resultados}

Dureza Shore D

Tabela 1. Dureza Shore D do PEAD com eletrodo de solda.

\begin{tabular}{ccc}
\hline Medida & CP1 (HSD) & CP2(HSD) \\
\hline $1^{\circ}$ & 61,4 & 62,9 \\
$2^{\circ}$ & 60,0 & 60,2 \\
$3^{\circ}$ & 61,2 & 60,4 \\
$4^{\circ}$ & 59,5 & 61,0 \\
$5^{\circ}$ & 61,4 & 60,2 \\
$6^{\circ}$ & 61,4 & 60,2 \\
\hline
\end{tabular}




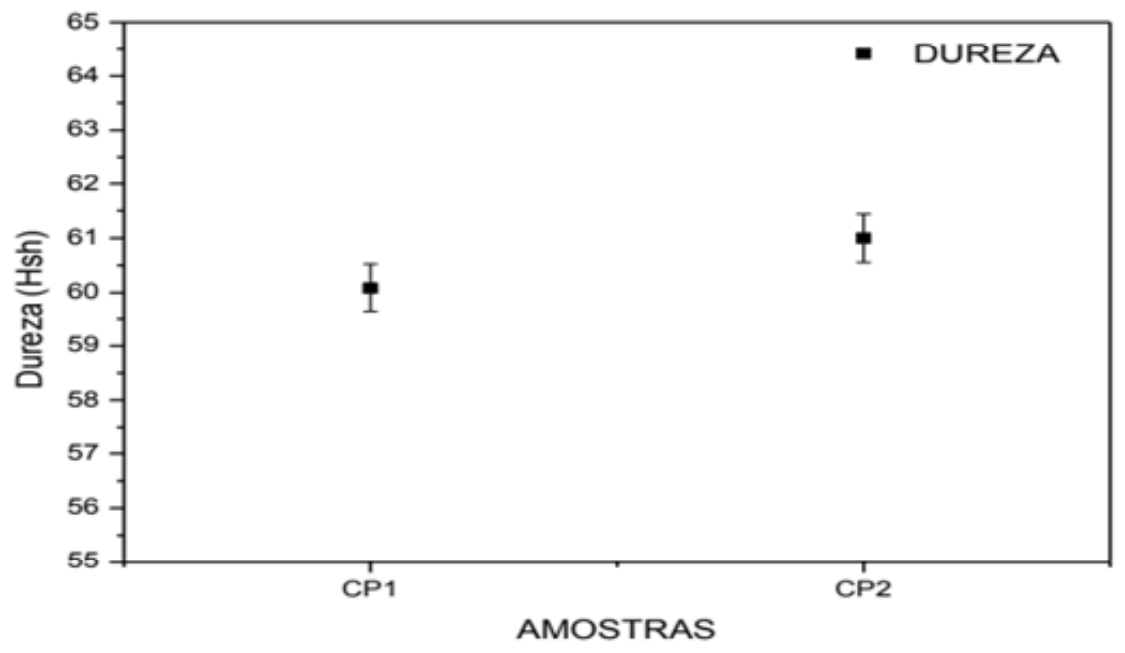

Figura 1. Resultados de ensaio de dureza Shore D.

Primeiramente foram realizados o ensaio de dureza nos corpos de prova submetidos ao ensaio de flexão. A tabela 1 mostra os resultados obtidos nas medições de dureza nos dois corpos de prova. Já na figura 1 pode-se observar os valores entre $60 \pm 0,444$ e $61 \pm 0,446$ Shore D, para o PEAD reforçado com eletrodo revestido (Loureiro, 2017).

\section{Resistência à flexão}

O ensaio de resistência à flexão foi realizado aplicando forças aos corpos de prova, sob condições controladas observando o material se flexionar. Para uma melhor observação dos resultados foram calculadas as médias desses valores que são apresentados na tabela

Tabela 2. Determinação da área útil dos corpos de provado PEAD com eletrodo de solda.

\begin{tabular}{cccc}
\hline $\begin{array}{c}\text { Corpo de } \\
\text { prova }\end{array}$ & Largura (nm) & Espessura (nm) & Área $\left(\mathrm{nm}^{2}\right)$ \\
& & & \\
\hline CP1 & 12,95 & 5,92 & 76,00 \\
CP2 & 12,95 & 5,95 & 77,00 \\
\hline Média & 12,95 & 5,935 & 76,50 \\
\hline
\end{tabular}




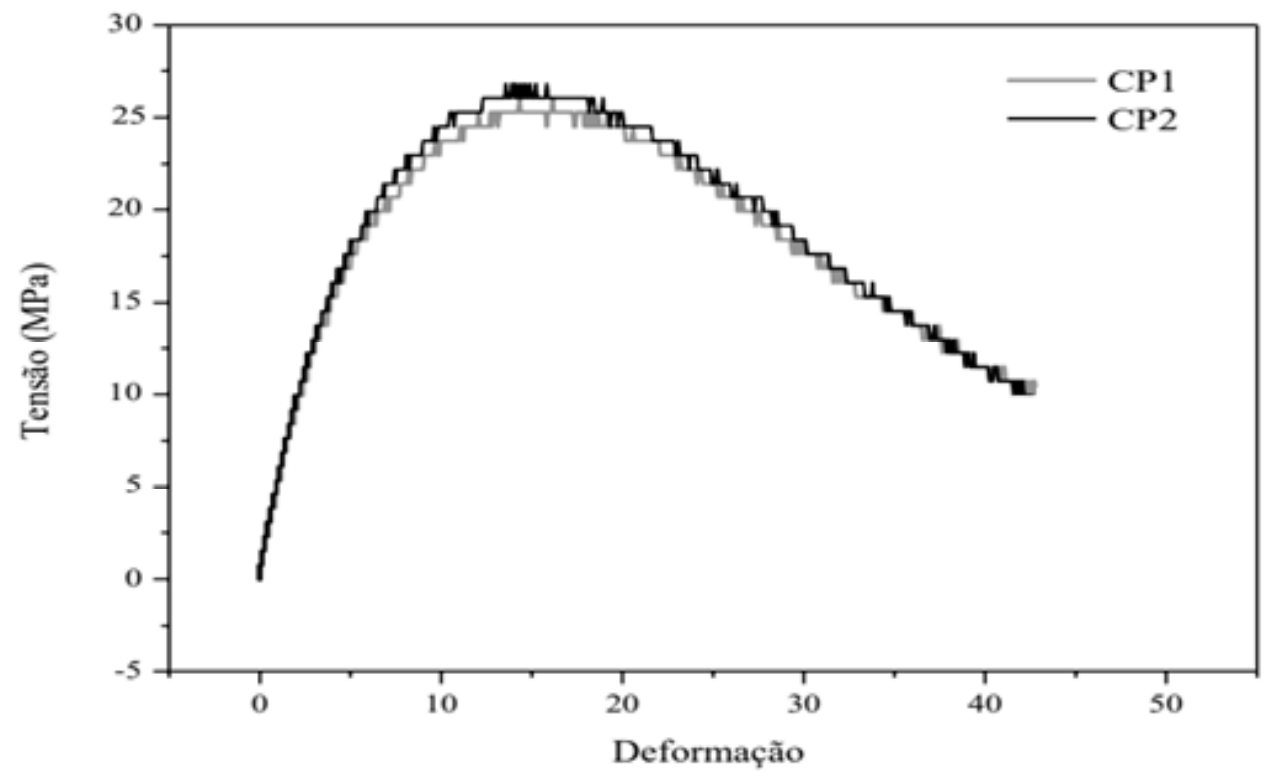

Figura 2. Resultado do ensaio de resistência à flexão.

\section{2 e Figura 2.}

\section{Microscopia de luz (óptica)}

As amostras embutidas foram analisadas no microscópio de luz (óptico), onde foram obtidas imagens que revelaram a fraca interação entre reforço e matriz já que o reforço pode ser claramente diferenciado da matriz. Na figura 3 (a),(b) e(c) fica evidente o que foi relatado anteriormente, onde as partículas também se encontram distribuídas de maneira desordenada.

\section{Microcopia Eletrônica de Varredura}

As imagens obtidas no MEV elucidam uma morfologia de superfície homogênea, e é possível

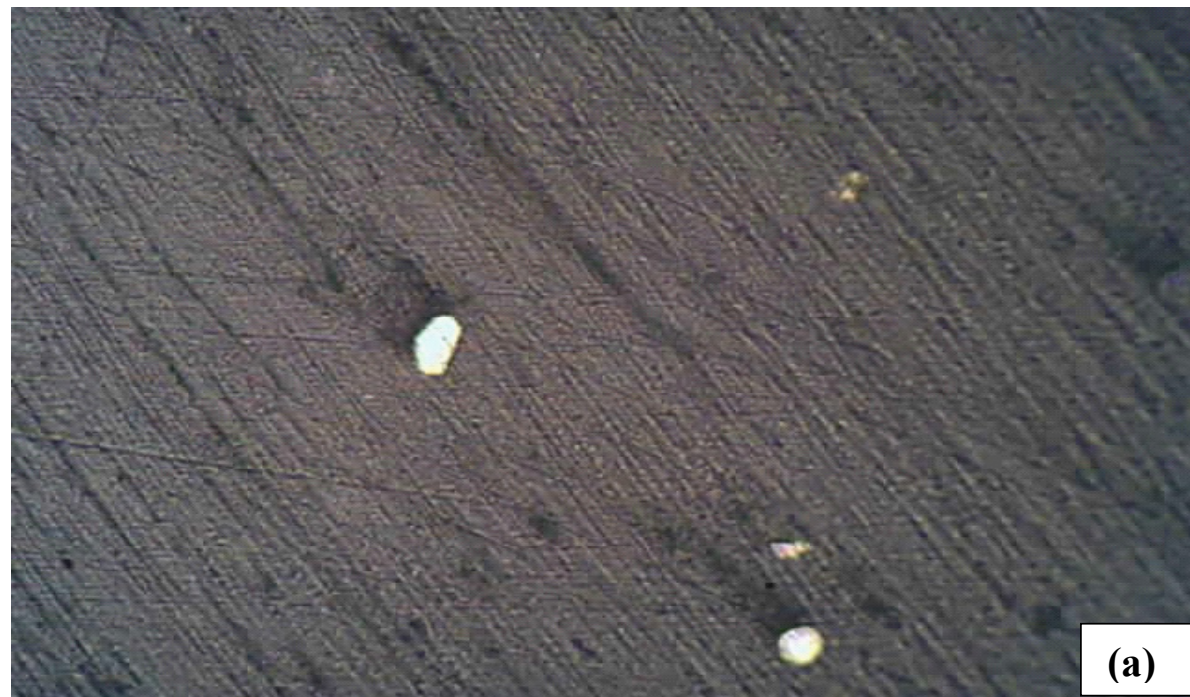



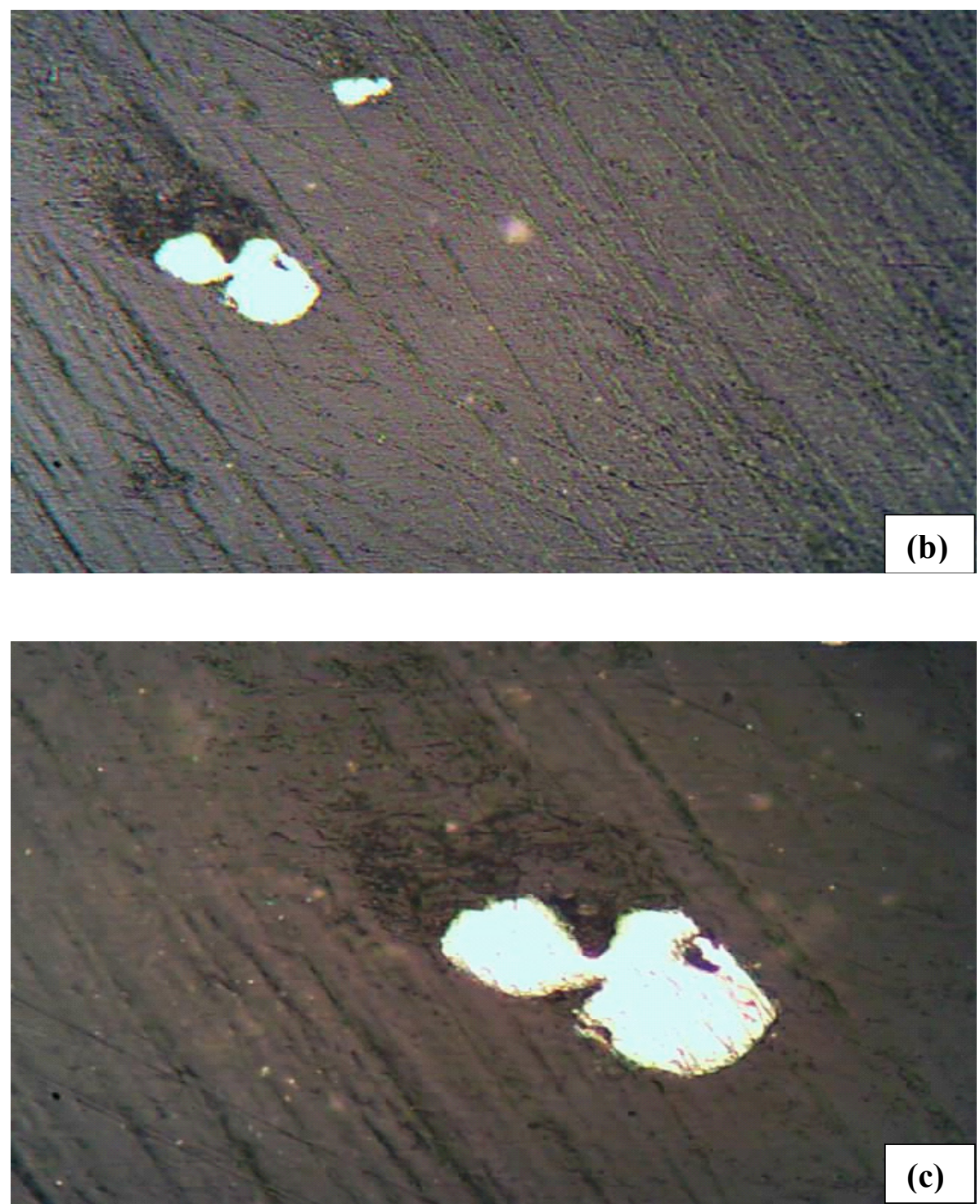

Figura 3. Microscopia óptica doPEAD com eletrodo de solda das amostras embutidas:(a) 1000X, (b) 2000X e (c) 4000X.

Fonte: autores, 2018.

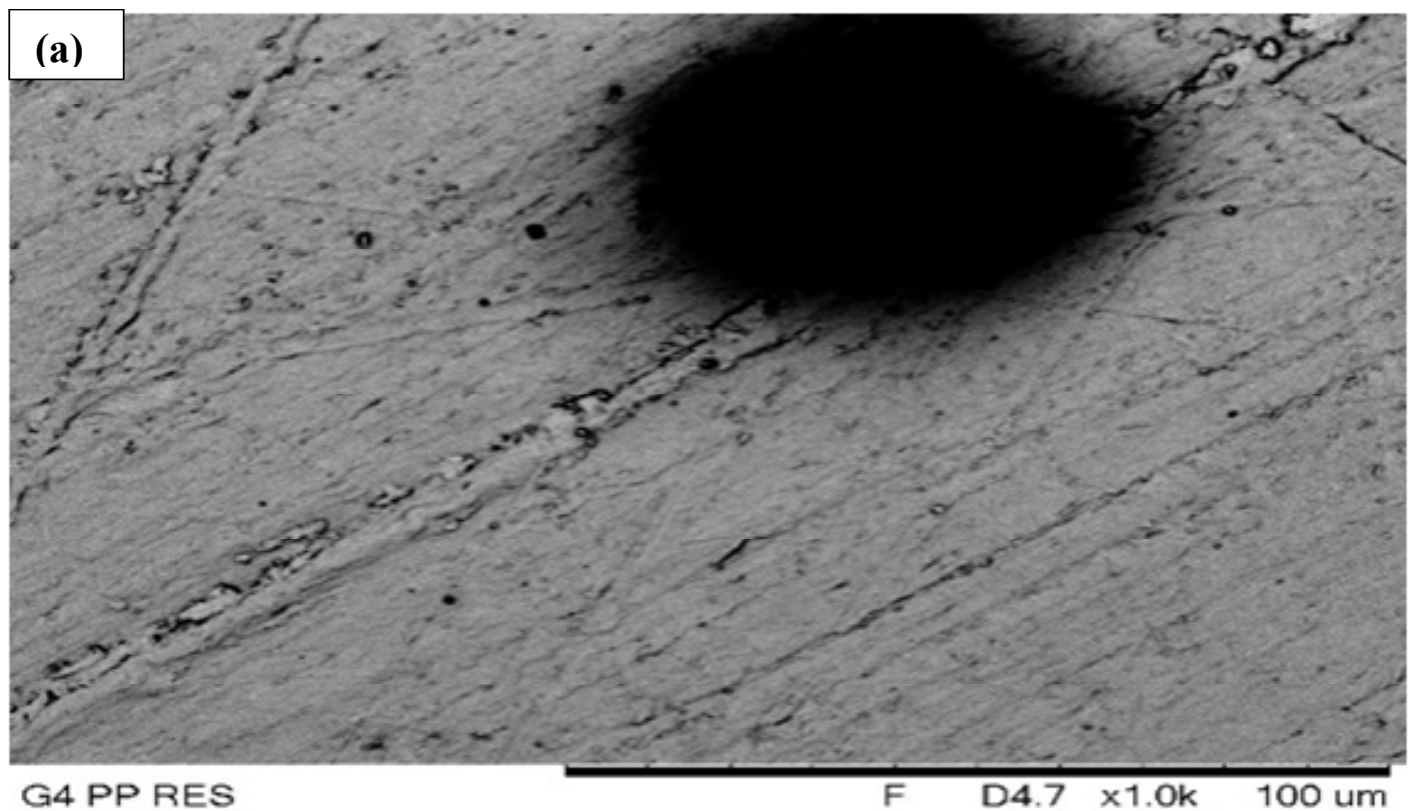



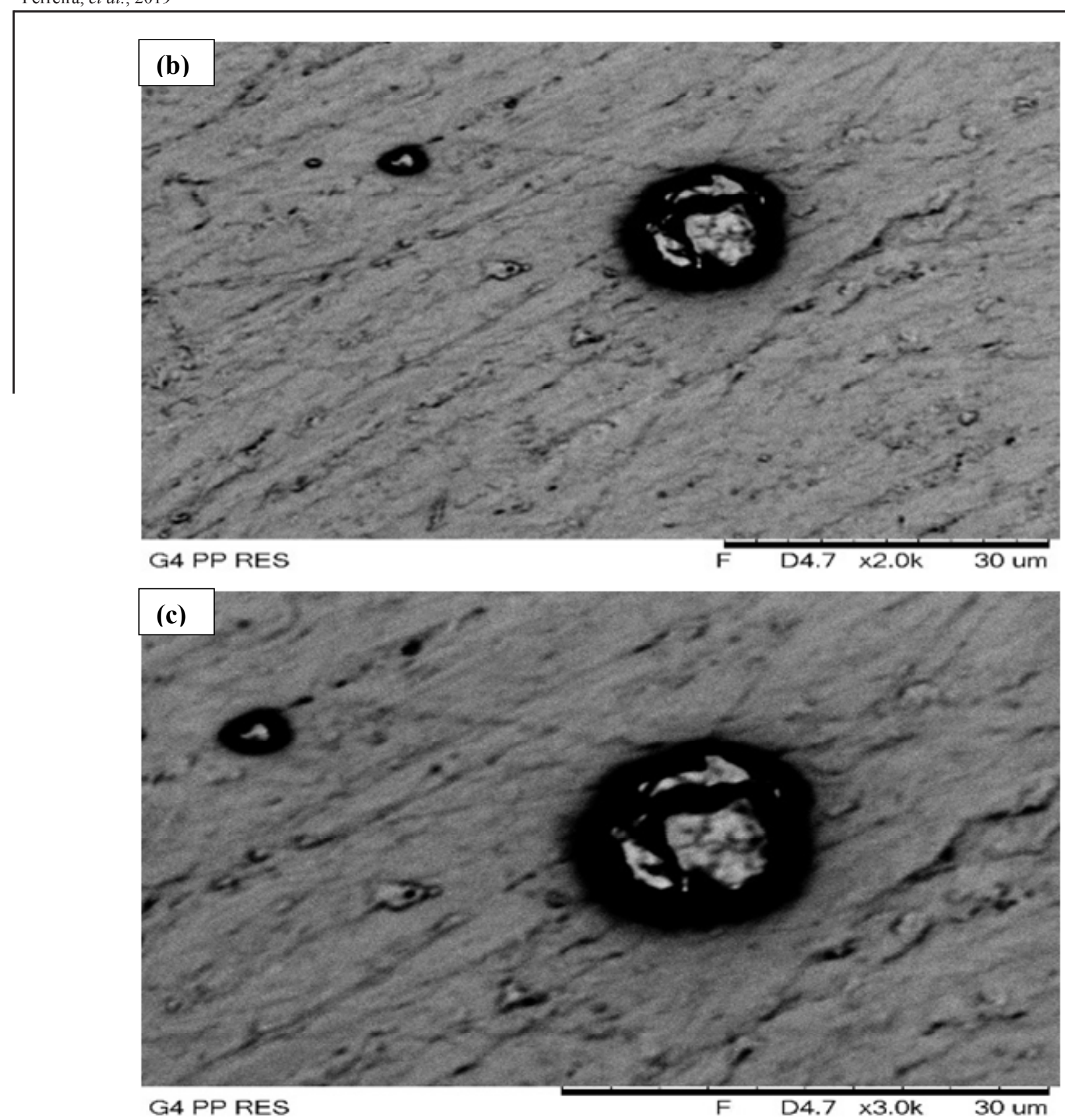

Figura 4. Imagens de MEV do PEAD com eletrodo de solda das amostras embutidas:(a) 1000X, (b) 2000X e (c) $3000 \mathrm{X}$.

Fonte: autores, 2018.

observar a diferença entre as amostras embutida e fraturada. Na figura 4 é possível identificar a amostra embutida com 1000X, 2000X e 3000X, em diferentes regiões.

\section{Discussões}

\section{Dureza shore}

De acordo com os estudos de Loureiro (2017), o ensaio de dureza Shore é realizado deixando uma impressão no corpo de prova, onde a penetração do mesmo é medida quando este é forçado sobre a amostra, sendo nesse estudo utilizado a dureza Shore D e observou-se a pouca alteração de dureza em relação ao PEAD, de acordo com a Tabela 1.

O PEAD tem como característica a realização de ligações chamadas de van der Waals o que causa ligações frágeis e altas chances de rompimento quando se introduz energia (Loureiro, 2017). Por esse motivo, nota-se na Figura 1 que os valores entre 60 e 61 Shore D para o PEAD reforçado com eletrodo, ficaram dentro dos parâmetros indicados na literatura.

\section{Resistencia à flexão}

Pode-se considerar a comparação de um ensaio de flexão com um determinado material quando o mesmo 

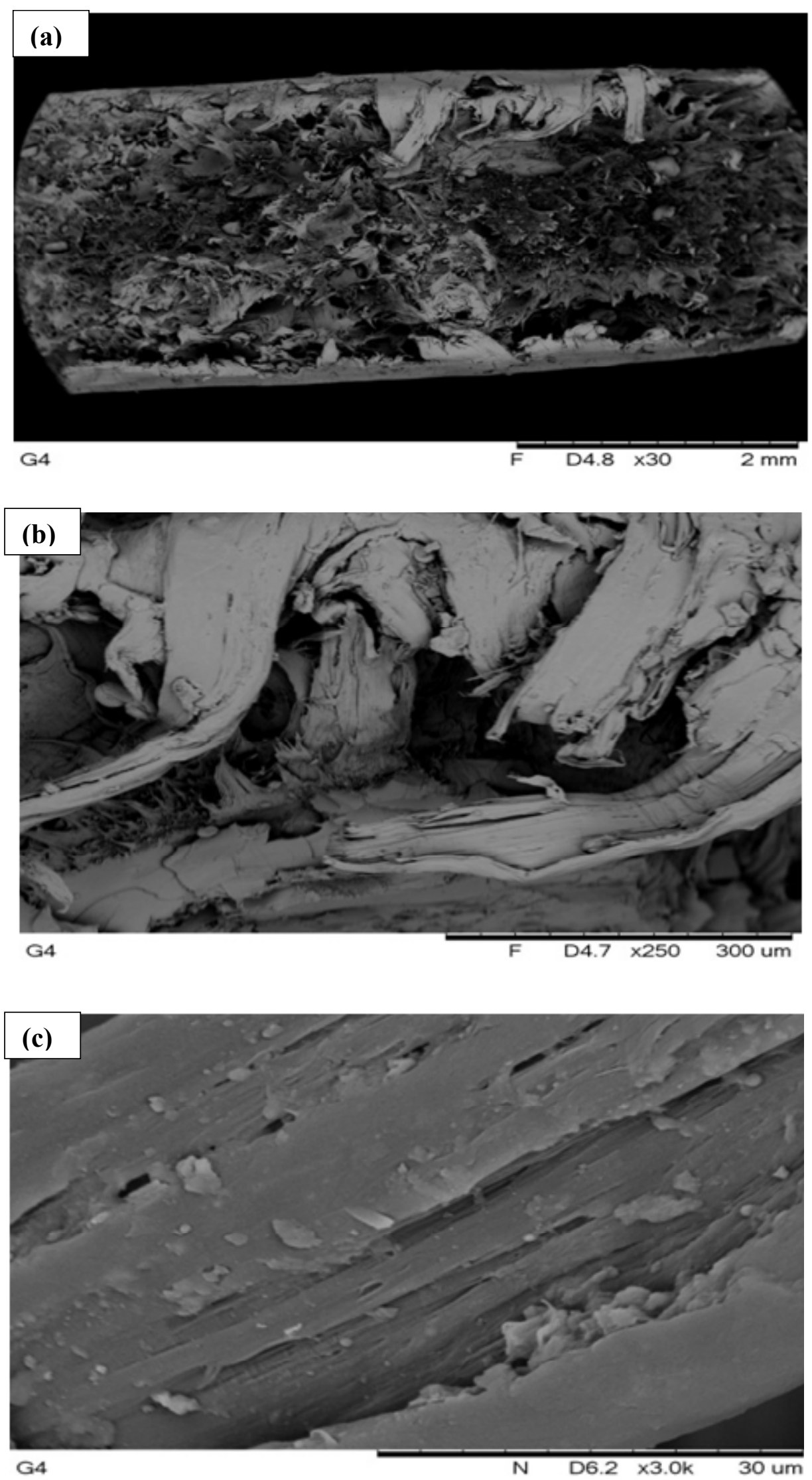

Figura 5. Imagens de MEV do PEAD com eletrodo de solda, das amostras fraturadas: (a) 30X, (b) $250 \mathrm{X}$ e (c) $3000 \mathrm{X}$.

Fonte: autores, 2018. 
é escorado como uma vida, considerando o corpo de prova apoiado em pontos marcados em uma distância medida e uma carga aplicada no meio considerado dessa distância em uma máquina universal de ensaios (Baptista, 2012).

A flexão segundo Loureiro (2017) em seus estudos de polímero reforçados com resíduos de sólidos do processo de soldagem a arco elétrico por eletrodos revestidos a partir de uma matriz de PEAD, no módulo de elasticidade, a flexão é diretamente influenciada pela quantidade de resíduo de eletrodo.

Sendo assim, os testes de flexão no estudo foram aplicados nos corpos de prova com a intenção de determinar o módulo de elasticidade e módulo de falhas a fim de avaliar as propriedades mecânicas, tensão e flecha de ruptura além do módulo de elasticidade. Após as realizações os valores das propriedades obtidos com os testes são representados na Figura 2 em curvas do tipo tensão versus deformação para o ensaio de flexão.

\section{Microscopia de luz (óptica)}

A partir da Microscopia óptica foi possível observar que as imagens revelaram a fraca interação entre o reforço e a matriz no material, o que pode ser observado nas figuras $3(a, b, c)$. As imagens revelaram que as partículas se encontraram desordenadas, e o material final foi um material homogênio.

\section{Microcopia Eletrônica de Varredura}

As imagens da figura 4 revelam a fraca interação entres as fases, o que já era esperada devido ao caráter orgânico da matriz e caráter inorgânico do reforço. Essas diferenças físico-químicas explicam essa falta de ou pouca interação (Coelho, 2013).

Nas imagens referentes a região fraturada, como mostrado na figura 5 , pode-se observar que o material tem comportamento plástico na fratura, podendo-se concluir que compósito de PEAD com eletrodo de solda aumenta a plasticidade do material e reduz a sua elasticidade. Esse comportamento plástico na fratura fica evidente no centro da primeira imagem da figura 5 . As outras imagens também revelam que apesar de um aumento na sua característica plástica o material ainda preserva o caráter elástico em algumas regiões de fratura. Também, ficou claro que na amostra fraturada, a pouca interação entre as fases está evidente. Para um melhor resultado de interação entre as fases, seria necessário a avaliação do uso de um agente compatibilizante (Coelho, 2013).

\section{Considerações finais}

Foi possível notar que, mesmo com reforço de eletrodo de solda, o material compósito não teve alterações com relação a dureza Shore D e se mantiveram entre 60 e 61 Shore D.

A microscopia óptica mostrou que o material não obteve boa interação entre o eletrodo de solda e polímero PEAD, com partículas desordenadas.

Já no MEV foi observada a diferença entre a amostra embutida e a fraturada. Verificou-se que as partículas de ferro contidas nos compósitos estão dispersas na matriz polimérica e as fibras, e as fases interagem fracamente.

\section{Referências Bibliográficas}

ASTM, D638-14 (2014). Test Method for TensilePropertiesofPlastics.

Baptista, C. (2012). Ensaios Mecânicos - Ensaios de Flexão. EEL - Escola de Engenharia de Lorena - Universidade de São Paulo.

Callister Jr, W. D., \& Rethwisch, D. G. (2012). Ciências e engenharias de Materiais: uma introdução. ( $\left.8^{\circ} \mathrm{ed}\right)$. Rio de Janeiro: Livros Técnicos e Científicos Editora LTDA.

Canevarolo, S. V. (2007). Técnicas de caracterização de polímeros. São Paulo: Artliber.

Coelho, P. H. S. L. \& Morales, A. R. (2013). Efeito da MontmorilonitaOrganifílica na Compatibilização e nas Propriedades Morfológicas, Mecânicas e Térmicas de Blendas de PA6/ PEBD. Revista Polimeros, 23 (3), 410-416.

Loureiro, A.T. (2017). Desenvolvimento e caracterização de compósitos de PEAD reforçados com resíduos de eletrodos revestidos.(Dissertação de Mestrado Profissional em Materiais, Fundação Oswaldo Aranha, 2017), 83, 4316.

Moreira, W. (2008). Compósitos 1 - Materiais, Processos, Aplicações, Desempenhos e Tendências. São Paulo: ALMAÇO Controle de qualidade na moldagem com resinas poliéster e gel coats, (2), 123-178.

Mano, E. B. \& Mendes, L.C. (1999). Introdução à polímeros. (2 ed.). São Paulo: Edgard Blucher. 